\title{
sciendo
}

\section{ENHANCEMENT OF IN VITRO DEVELOPMENTAL OUTCOME OF CLONED GOAT EMBRYOS AFTER EPIGENETIC MODULATION OF SOMATIC CELL-INHERITED NUCLEAR GENOME WITH TRICHOSTATIN A*}

\author{
Maria Skrzyszowska ${ }^{+}$Marcin Samiec ${ }^{1}$ \\ Department of Reproductive Biotechnology and Cryoconservation, \\ National Research Institute of Animal Production, 32-083 Balice n. Kraków, Poland \\ •Corresponding author: maria.skrzyszowska@izoo.krakow.pl
}

\begin{abstract}
In this study, the effect of trichostatin A (TSA)-mediated epigenomic modulation of nuclear donor cells on the in vitro developmental potential of caprine somatic cell cloned embryos was examined. The enucleated ex vivo-matured oocytes were subzonally injected with adult ear skin-derived fibroblast cells exposed or not exposed to TSA (at a concentration of $50 \mathrm{nM}$ ). The experiment was designed on the basis of three different approaches to TSA-dependent modulation of donor celldescended genome: before being used for somatic cell nuclear transfer/SCNT (Group I); immediately after activation of nuclear-transferred (NT) oocytes (Group II); or combined treatment both before being used for SCNT and after activation of NT oocytes (Group III). In the control Group IV, donor cell nuclei have not been treated with TSA at any stage of the experimental design. In TSA-treated Groups I and II and untreated Group IV, cleavage activities of cloned embryos were at the similar levels $(\mathbf{8 0 . 6} \%, \mathbf{7 9 . 8} \%$ and $77.1 \%$, respectively). But, significant difference was observed between Groups III and IV (85.3 vs. $77.1 \%)$. Moreover, in the experimental Groups I and III, the percentages of cloned embryos that reached the blastocyst stages remarkably increased as compared to those noticed in the control Group IV (31.2\% vs. 36.7\% vs. $18.9 \%$, respectively). In turn, among embryos assigned to Group II, blastocyst formation rate was only slightly higher than that in the control Group IV, but the differences were not statistically significant $(25.8 \%$ vs. $18.9 \%$ ). To sum up, TSA-based epigenomic modulation of somatic cell-inherited nuclear genome gave rise to increased competences of caprine cloned embryos to complete their development to blastocyst stages. In particular, sequential TSA-mediated modulation of both nuclear donor cells and activated NT oocytes led to improvement in the blastocyst yields of cloned goat embryos, which can result from enhanced donor cell nuclear reprogrammability.
\end{abstract}

Key words: goat, donor cell, trichostatin A, somatic cell nuclear transfer, cloned embryo

\footnotetext{
* Presented work was financially supported by the National Centre for Research and Development in Poland (grant number BIOSTRATEG2/297267/14/NCBR/2016).

${ }^{1}$ Maria Skrzyszowska and Marcin Samiec contributed equally both to this research and to the preparation of this paper.
} 
The efficiency of somatic cell nuclear transfer (SCNT) in goats, as measured by rate of the cloned progeny born, is still relatively low and generally fails to exceed an average of $10 \%$ in relation to the number of cloned embryos transferred into the reproductive tracts of recipient does, and 5\% in relation to the number of nuclear-transferred (NT) oocytes (Martins et al., 2016; Deng et al., 2017; Lu et al., 2018; Fernandes et al., 2018). Abnormal adaptation of transferred somatic cell nuclei to biochemical conditions of the oocyte cytoplasmic environment (and as a consequence, the fact that they are incompletely or aberrantly reprogrammed in the descendant blastomeres of SCNT-derived embryos) seems to be the main reason of both low pre- and postimplantation developmental potential of mammalian cloned embryos and their diminished quality (Eilertsen et al., 2007; Jia et al., 2017; Zuo et al., 2017; Zhang et al., 2018; Samiec and Skrzyszowska, 2018 a, b). So far, many investigators have undertaken research to explain the mechanisms underlying capabilities of somatic cell nuclei to be efficiently reprogrammed (i.e., dedifferentiated) in the host cytoplasm of NT oocytes (Rodriguez-Osorio et al., 2012; Huan et al., 2015; Sepulveda-Rincon et al., 2016; Jin et al., 2017; Kumar and Sarkhel, 2017).

Wan et al. (2016) have shown that, in cloned goat-derived fibroblast cells, the processes of abnormal DNA methylation within differentially methylated regions (DMRs) of $H 19$ and $\operatorname{Ig} f 2 r$ genes that are found to result from loss of abundant quantitative profiles of DNMT enzymes turned out to be the consequences of aberrant epigenetic nuclear reprogramming during SCNT. It should also be noted that improper transcriptional activity (i.e., upregulation or downregulation) of not only several pluripotency-related genes (Oct4, Nanog), but also genes encoding insulin-like growth polypeptide factors and their receptors $(\operatorname{Ig} f 2, \operatorname{Ig} f 2 r)$ and DNMT isozymes (Dnmt1, Dnmt3a, Dnmt3b) was confirmed in caprine and other mammalian species cloned embryos (Huan et al., 2015; Deng et al., 2017; Kumar and Sarkhel, 2017; Jia et al., 2017). As a consequence, aberrant or incomplete reprogramming of donor cell nuclei has brought about declined incidences for blastocyst formation rate and pregnancy establishment as compared to those noticed for the in vitro- and in vivoproduced embryos (Chies et al., 2016; Martins et al., 2016; Kumar and Sarkhel, 2017; Zuo et al., 2017).

Histone deacetylases (HDACs) and DNA methyltransferases (DNMTs) have been found to be indispensable for suppressing transcriptional activity of the genes by biocatalysing deacetylation processes within chromatin nucleosomal core-derived histone lysine moieties and methylation processes within cytosine residues of nuclear DNA, respectively. These processes lead to diminishment in epigenetic reprogrammability of somatic cell nuclei in activated mammalian NT oocytes and resultant cloned embryos (Sepulveda-Rincon et al., 2016; Liu et al., 2018; Samiec and Skrzyszowska, 2018 a, b). Due to aberrant or incomplete epigenetic reprogramming of donor cell nuclei in a cytoplasm of NT oocytes and resultant cloned embryos, the low efficiency of somatic cell cloning in mammals limits the practical application of this assisted reproductive technology for the purposes of biomedicine and agriculture (Samiec, 2004; Loi et al., 2016; Samiec and Skrzyszowska, 2010, 2011; Fan et al., 2019). The main efforts to improve the effectiveness of SCNT procedure seem to be 
focused on the use of non-specific HDAC and/or DNMT inhibitors for epigenomic modulation (i.e., epigenetic transformation) of not only ex vivo expanded nuclear donor cells, but also in vitro maturing nuclear recipient oocytes or activated NT oocytes (Samiec and Skrzyszowska, 2012; Sangalli et al., 2014; Wen et al., 2014; Gupta et al., 2019; Samiec et al., 2019).

Varied approaches have been recently accomplished to use extrinsic compounds (i.e., non-selective inhibitors of HDACs and/or DNMTs) for epigenomic modulation of nuclear donor cell-inherited genome, which have brought about not only facilitating its reprogrammability in mammalian cloned embryos, but also improving cytological and molecular quality of the blastocysts generated (Saini et al., 2014; Samiec et al., 2015; Agrawal et al., 2018). Treatment of in vitro cultured somatic cells and/ or activated NT oocytes either with a broad spectrum of non-specific HDAC and/ or DNMT inhibitors, among others trichostatin A (TSA), belinostat (PXD101), dacinostat (LAQ824), valproic acid (VPA), scriptaid (SCPT), 5-aza-2'-deoxycytidine (5-aza-dC) or with a selective class I HDAC inhibitor known as tacedinaline (CI994), has contributed to a considerable increase in the blastocyst formation rates of cloned embryos in different mammalian species, including mice (Kishigami et al., 2006; Qiu et al., 2017), pigs (Jin et al., 2017, 2018), cattle (Ding et al., 2008; Sangalli et al., 2014), sheep (Wen et al., 2014) and buffalos (Saini et al., 2017).

Furthermore, Saini et al. (2014) have demonstrated that TSA-mediated epigenomic modulation improves in vitro developmental competences and quality of bovine cloned embryos by regulating the expression of microRNAs specific for G0/G1 stages of mitotic cycle in nuclear donor cells. As a consequence, these investigators have proved that TSA can exhibit not only properties of potent epigenetic modulator but also it can play an important role as a pivotal cell cycle synchronizer for the purposes of SCNT research. Additionally, comprehensive research by Hosseini et al. (2016) has shown that TSA-dependent epigenomic modulation of nuclear donor cells does not evoke the cessation/erasure or reversal of somatic cell genome site/sequence-specific and oocyte-mediated reprogramming errors triggering non-random aberrations (upregulation or downregulation) in transcriptomic activity throughout development of bovine SCNT-derived embryos. In spite of that, treatment of bovine nuclear donor cells with TSA has led to improvements both in the in vitro developmental potential and in the molecular quality of cloned embryos as compared to SCNT-derived embryos produced using TSA-untreated somatic cells.

Therefore, the aim of this study was to determine the effect of epigenomic modulation of somatic cell-derived nuclear genome on the in vitro developmental competences of caprine cloned embryos. Furthermore, in the present investigation, TSA (a non-selective HDAC inhibitor at a concentration $50 \mathrm{nM}$ ) that stimulates epigenetically regulated transcriptional activity of genomic DNA both in nuclear donor cells (NDCs) and in activated NT oocytes or only in activated NT oocytes was used for the purposes of generating cloned goat embryos for the first time. 


\section{Material and methods}

\section{Source of chemical purchase and bioethical guidelines}

All reagents were purchased from Sigma-Aldrich Corporation (Poznań, Poland) being a part of Merck Group (Merck KGaA, Darmstadt, Germany), unless otherwise stated. The procedures involving collection of skin bioptates and oocytes were approved by the Local Institutional Animal Care and Use Committee in Kraków (Poland) according to animal welfare laws, guidelines and policies.

\section{Preparation of ear skin-derived fibroblast cells}

In the somatic cell cloning procedure, dermal fibroblast cells from a few-weekold kids were used as nuclear donors. Primary cell cultures were established from ear skin tissue-derived explants. Subcutaneous and cutaneous bioptates were subjected to mechanical disaggregation, and small tissue sections were trypsinized to disperse the cells. Cell cultures were accomplished in Dulbecco's Modified Eagle's Medium (DMEM, Gibco BRL, Life Technologies Ltd., Poznań, Poland) supplemented with 2 mM L-glutamine, 1\% Modified Eagle's Medium-non-essential amino acid solution (MEM-NEAA) and 10\% foetal bovine serum (FBS). Somatic cell lines were passaged after reaching a total confluence, i.e., after the bottom of a culture flask had been completely covered by adherent cell monolayer. Fibroblast cell lines underwent artificial synchronization of the mitotic cycle at the G0/G1 phases by contact inhibition of migration and proliferative growth under a total confluence. Before their use for SCNT, ex vivo expanded fibroblast cells were epigenomically modulated by incubation in the medium supplemented with $50 \mathrm{nM}$ TSA for 23 to $24 \mathrm{~h}$. TSA-treated or untreated cells that had been intended to be nuclear donors were selected for SCNT procedure.

\section{Collection and in vitro meiotic maturation of nuclear recipient oocytes}

Immature cumulus-oocyte complexes (COCs) were recovered by laparoscopic ovum pick-up (LOPU) of follicular fluid from ovarian antral follicles. LOPU-mediated oocyte retrieval was performed through abdominal integuments of anaesthetized donor does, whose oestrus cycle had been synchronized by administering a synthetic progesterone analogue-impregnated intravaginal sponges containing fluorogestone acetate (FGA, Chronogest CR, Intervet, Angers, France). Subsequently, oestrus-synchronized females were hormonally stimulated with Ovagen, i.e., follicle-stimulating hormone (FSH) extracted from ovine pituitary glands (ICPbio Ltd., Auckland, New Zealand). The quality of caprine COCs was assessed on the basis of the presence and appearance of surrounding cumulus oophorus cells and ooplasm homogeneity. Morphologically normal oocytes that displayed an evenly granulated cytoplasm not undergoing degenerative (necrotic or apoptotic) changes and were enclosed within at least three compact layers of cumulus cells were selected for in vitro maturation (IVM). Caprine COCs were cultured for 23 to $24 \mathrm{~h}$ in IVM medium that was comprised of Tissue Culture Medium 199 (TCM 199) enriched with 20\% heat-inactivated oestrus goat serum (EGS), $1 \mu \mathrm{g} / \mathrm{mL} 17-\beta$-oestradiol, $5 \mathrm{mU} / \mathrm{mL}$ porcine FSH and $10 \mathrm{ng} / \mathrm{mL}$ recombinant human epidermal growth factor (rhEGF). In vitro-matured 
oocytes whose meiotic cycle was transiently arrested at the metaphase II (MII) stage were characterized by the occurrence of both homogeneously granulated cytoplasm and distinctly extruded first polar bodies and provided a source of recipient cells for somatic cell nuclei in the cloning procedure.

\section{Generation of caprine embryos by SCNT}

\section{Chemically-assisted enucleation of oocytes}

In vitro-matured oocytes deprived of cumulus cells were incubated for 45 to $60 \mathrm{~min}$ in TC 199 medium supplemented with $0.4 \%$ bovine serum albumin (BSA), $0.4 \mu \mathrm{g} / \mathrm{mL}$ of demecolcine (DMCC) and $0.05 \mathrm{M}$ sucrose. Fifteen minutes before oocyte enucleation, a dose of $7.5 \mu \mathrm{g} / \mathrm{mL}$ cytochalasin B was added to DMCC- and sucrose-enriched medium. Afterwards, the first polar body and the adjacent ooplasmic protrusion (containing MII chromosomes) were microsurgically removed with the aid of a beveled glass pipette, whose external diameter ranged from 15 to $20 \mu \mathrm{m}$.

\section{Reconstruction of enucleated oocytes}

Single nuclear donor cells were microinjected under the zonae pellucidae of enucleated oocytes (ooplasts) in such a manner that the somatic cell plasma membrane adhered tightly to the ooplast plasma membrane. The resulting somatic cell-ooplast couplets (SCOCs) were placed in an electrofusion chamber filled with a dielectric solution (0.3 M mannitol supplemented with $0.05 \mathrm{mM} \mathrm{CaCl}_{2}, 0.1 \mathrm{mM} \mathrm{MgSO}_{4}$, and $0.2 \mathrm{mg} / \mathrm{mL}$ fatty acid-free BSA). Electrofusion of SCOCs was triggered by single direct current (DC) pulse of $2.4 \mathrm{kV} / \mathrm{cm}$ for $15 \mu \mathrm{s}$ that had been generated by a BTX Electrocell Manipulator 200 (Biotechnologies \& Experimental Research Inc., San Diego, USA). The successfully fused SCOCs were incubated in synthetic oviductal fluid (SOF) medium for 1.5 to $2 \mathrm{~h}$ at $38.5^{\circ} \mathrm{C}$, in an atmosphere of $90 \% \mathrm{~N}_{2}, 5 \% \mathrm{O}_{2}$ and $5 \% \mathrm{CO}_{2}$ with a water-saturated humidity.

\section{Artificial activation of reconstructed oocytes}

After a 1.5- to 2-h delay post reconstruction, nuclear-transferred oocytes (clonal cybrids) were activated by their exposure to $5 \mu \mathrm{M}$ calcium ionomycin for $5 \mathrm{~min}$ followed by 2-h incubation in SOF medium supplemented with $2 \mathrm{mM}$ 6-dimethyloaminopurine (6-DMAP) at $38.5^{\circ} \mathrm{C}$ in $5 \% \mathrm{CO}_{2} / \mathrm{O}_{2}$ in a humidified air.

\section{In vitro culture of caprine cloned embryos}

SCNT-derived embryos were rinsed thrice using fresh SOF medium and cultured in single wells of 4-well dishes filled with $500 \mu \mathrm{L}$ of SOF medium supplemented with or deprived of $50 \mathrm{nM}$ of TSA at $38.5^{\circ} \mathrm{C}$ in an atmosphere of $90 \% \mathrm{~N}_{2}, 5 \% \mathrm{O}_{2}$ and $5 \% \mathrm{CO}_{2}$ with a water-saturated humidity. After a 24-h incubation, cleaved embryos were placed in TSA-free SOF medium enriched with 10\% FBS and cultured for an additional of 5 to 6 days until they have reached the morula/blastocyst stages.

\section{Experimental design}

Three different approaches to TSA-mediated epigenomic modulation of donor cell nuclei have been used as follows: in the first approach (Group I) - before being 
used for cloning; in the second approach (Group II) - immediately after activation of NT oocytes; and in the third combined approach (Group III) - both before being used for cloning and immediately after activation of NT oocytes. Cloned embryos created using both TSA-untreated nuclear donor cells (NDCs) and TSA-untreated activated NT oocytes have been allotted to control Group IV.

\section{Statistical analysis}

All experiments were repeated at least three times. Developmental rates of caprine cloned embryos were analyzed by using $\chi^{2}$ test. The percentages of NT oocytes, cleaved embryos and the morulae and blastocysts generated were compared between experimental and control groups. The inter-group variability with the probability at the level of $P$ less than 0.05 was considered to be statistically significant.

\section{Results}

Data encompassing cleavage activities and morula/blastocyst formation rates of caprine cloned embryos generated either using three different approaches to TSAmediated epigenomic modulation of donor cell nuclei (Groups I, II and III) or using TSA-unexposed NDCs and TSA-unexposed activated NT oocytes (Group IV) have been presented in Table 1. In turn, Figure 1 depicts caprine cloned blastocysts produced by various approaches to epigenomic modulation of somatic cell nuclei.

A

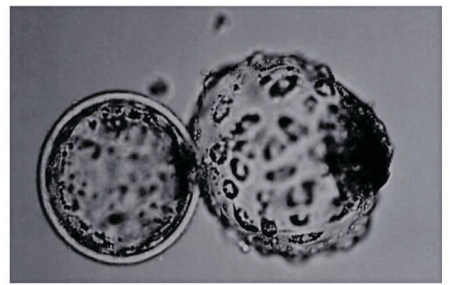

C

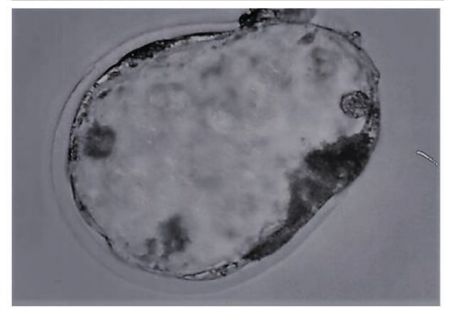

B

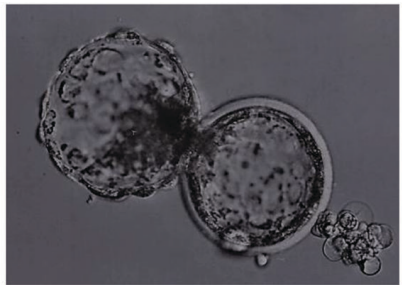

D

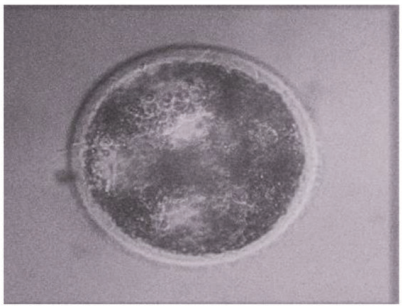

Figure 1. Caprine cloned blastocysts generated by different approaches to epigenomic modulation of donor cell nuclei. A) Blastocyst developed in vitro from nuclear-transferred (NT) embryo reconstructed with adult dermal fibroblast cell (ADFC) exposed to trichostatin A (TSA) only before being used for somatic cell cloning (SCC; Group I); B) Blastocyst developed from embryo reconstructed with ADFC nucleus exposed to TSA after activation of NT oocyte (Group II); C) Blastocyst developed from embryo reconstructed with ADFC nucleus exposed to TSA both before being used for SCC and immediately after activation of NT oocyte (Group III); D) Blastocyst developed from NT embryo reconstructed with TSA-untreated ADFC (Group IV). Original magnification $\times 200$ 
Table 1. Dependence of the in vitro developmental rates of caprine cloned embryos on various approaches to TSA-based epigenomic modulation of somatic cell nuclei

\begin{tabular}{l|c|c|c|c}
\hline \multicolumn{1}{c|}{ The approach to epigenomic modulation } & $\begin{array}{c}\text { Number } \\
\text { of NT oocytes } \\
(\%)\end{array}$ & $\begin{array}{c}\text { Number } \\
\text { of cleaved } \\
\text { embryos } \\
(\%)\end{array}$ & $\begin{array}{c}\text { Number } \\
\text { of morulae } \\
(\%)\end{array}$ & $\begin{array}{c}\text { Number } \\
\text { of blastocysts } \\
(\%)\end{array}$ \\
\hline TSA-treated NDCs & $93 / 117$ & $75 / 93$ & $44 / 93$ & $29 / 93$ \\
(Group I) & $(79.5)$ & $(80.6)$ & $(47.3) \mathrm{aA}$ & $(31.2) \mathrm{A}$ \\
TSA-treated activated NT oocytes & $89 / 106$ & $71 / 89$ & $49 / 89$ & $23 / 89$ \\
(Group II) & $(84.0)$ & $(79.8)$ & $(55.1) \mathrm{C}$ & $(25.8) \mathrm{a}$ \\
TSA-treated NDCs and activated NT oocytes & $109 / 135$ & $93 / 109$ & $62 / 109$ & $40 / 109$ \\
(Group III) & $(80.7)$ & $(85.3) \mathrm{a}$ & $(56.9) \mathrm{bC}$ & $(36.7) \mathrm{bC}$ \\
TSA-untreated NDCs and activated NT oocytes & $227 / 270$ & $175 / 227$ & $80 / 227$ & $43 / 227$ \\
(Group IV) & $(84.1)$ & $(77.1) \mathrm{b}$ & $(35.2) \mathrm{B}$ & $(18.9) \mathrm{B}$ \\
\hline
\end{tabular}

Absence of small letters behind the values within the same column denotes the lack of statistically significant differences between experimental groups ( $\mathrm{P} \geq 0.05 ; \chi^{2}$ test).

Presence of various small letters ( $\mathrm{a}$ and $\mathrm{b}$ ) behind the values within the same column denotes the occurrence of significant differences between experimental groups $\left(\mathrm{P}<0.05 ; \chi^{2}\right.$ test $)$.

Presence of various capital letters (A and B) behind the values within the same column denotes the occurrence of highly significant differences between experimental groups $\left(\mathrm{P} \leq 0.01 ; \chi^{2}\right.$ test $)$.

Presence of various capital letters (B and C) behind the values within the same column denotes the occurrence of very highly significant differences between experimental groups $\left(\mathrm{P}<0.001 ; \chi^{2}\right.$ test $)$.

Abbreviations: TSA - trichostatin A; NDCs - nuclear donor cells; NT - nuclear-transferred.

TSA treatment of activated NT oocytes (Group II) resulted in the cleavage activities that did not vary significantly from those noticed for Groups I and Group IV $(\mathrm{P} \geq 0.05)$. However, regardless of the approach to TSA-based epigenomic modulation of somatic cell-inherited nuclear genome (Groups I and III), the competences of cloned embryos to complete their development to morula stage turned out to be significantly (approximately one-and-a-half times) higher than those observed in the TSA-untreated Group IV. It is also worth highlighting that there were highly significant differences in the blastocyst yields $(\mathrm{P}<0.01)$ between cloned embryos generated using TSA-treated nuclear donor cells (Group I) and their counterparts generated using TSA-untreated nuclear donor cells (Group IV). Even (i.e., almost twofold) higher statistical variability in the blastocyst formation rates $(\mathrm{P}<0.001)$ was identified between cloned embryos derived from both TSA-exposed NDCs and TSAexposed activated NT oocytes (Group III) and their counterparts originating from TSA-untreated NDCs and TSA-untreated activated NT oocytes (Group IV).

\section{Discussion}

The results of our present study have confirmed that TSA significantly affects the ex vivo developmental capability of cloned goat embryos to reach the morula and blastocyst stages. So far, TSA has been utilized for modifying epigenetic status of cloned embryos in different mammalian species, including laboratory animals and livestock, and appears to be not only one of the most widely recognized and preva- 
lent, but also the most efficient and the least cytotoxic or the least embryotoxic members of the family of non-selective HDAC inhibitors (Samiec et al., 2015; Hosseini et al., 2016; Qiu et al., 2017). However, up to now, there have been no reports that assess the role of TSA either in epigenomic modulation of both caprine nuclear donor cells and SCNT-derived embryos (following artificial activation of reconstructed oocytes) or in epigenomic modulation of only activated caprine NT oocytes. We have chosen a concentration of $50 \mathrm{nM}$ TSA for epigenomic modulation of caprine NDCs and/or activated NT oocytes, because applying the above-mentioned dose of TSA to epigenetic transformation of somatic cell nuclei turned out to be highly efficient in our previous studies focused on producing intra- and intergeneric cloned embryos in other livestock species such as pigs and cattle (Samiec and Skrzyszowska, 2012; Samiec et al., 2015, 2019; Opiela et al., 2017).

In our current investigation, the percentage of caprine SCNT-derived embryos that developed in vitro to morula and blastocyst stages did not differ considerably between three experimental approaches applied to TSA-dependently epigenetically transforming nuclear genome of somatic cells as follows: 1) before being used for SCNT; 2) immediately after activation of NT oocytes; and 3) before being used for SCNT and after activation of reconstructed oocytes. Nevertheless, highly significant differences in the morula/blastocyst formation rates have been identified as compared to TSA-untreated group. It is also worth noting that TSA-dependent epigenomic modulation of only activated NT oocytes has led to obtaining the percentage of blastocysts that was similar to such percentage observed in a control group. It seems to be a quite surprising result, taking into consideration the fact that the morula yield in this group varied remarkably from that noticed for a control group. Lack of TSA-based epigenomic modulation of nuclear donor cells before their use for SCNT has given rise to a drastic diminishment of blastocyst formation rate in this experimental group. This clearly indicates that TSA-mediated modulation of donor cells (before being utilized for SCNT) can be crucial to enhancement of susceptibility of somatic cell nuclei to epigenetic reprogramming in a host cytoplasm of activated NT oocytes and descendant blastomeres of developing cloned goat embryos. The above-mentioned finding appears to trigger facilitating and/or accelerating onset of epigenetically regulated transcriptional activity of somatic cell-inherited genomic DNA. Furthermore, such finding is compatible with the conclusions resulting from the study by Mao et al. (2018). These investigators have demonstrated that treatment of only nuclear donor cells with a specific inhibitor of lysine-specific demethylase 1 (LSD1), designated as trans-2-phenylcyclopropylamine (2-PCPA), gives rise strikingly to improvement in the in vitro developmental competences of caprine cloned embryos to reach the morula and blastocyst stages. It is beyond any doubt that 2-PCPA-dependent epigenomic modulation has promoted upregulation of quantitative profiles specific for histone $\mathrm{H} 3 \mathrm{~K} 4$ dimethylation, which triggered enhancements in epigenetic reprogrammability and transcriptional activity of somatic cell-inherited nuclear genome during development of SCNT-derived goat embryos (Mao et al., 2018).

Similar to the results of our study, the beneficial effect of TSA-dependent epigenomic modulation of nuclear donor cells and/or activated NT oocytes on the in vitro development of cloned embryos was reported in goats and other ruminant species. 
Wang et al. (2015) have confirmed that epigenetic transformation of only nuclear donor fibroblast cells by their 12-h exposure to $50 \mathrm{nM}$ TSA biased improvements in the ex vivo capacity of SCNT-derived goat embryos to complete their development to the blastocyst stage at the percentage of $29.2 \%$. This percentage turns out to be similar to that noticed in our current research (31.2\%) following 23- to 24-h epigenomic modulation of caprine fibroblast cells with the use of identical concentration of TSA. In cattle, Wang et al. (2011) applied a sequential co-treatment of nuclear donor cells and early SCNT-derived embryos with 5-aza-dC and TSA, which brought about the significant increase in the blastocyst formation rates as compared to those indicated for 5-aza-dC/TSA-untreated group. As has been shown in the studies by Ding et al. (2008) and Iager et al. (2008), due to inhibiting DNA methylation and histone deacetylation, 5-aza-dC and TSA co-treatment causes enhancements in the occurrence of both demethylation processes within genomic DNA cytosine residues and histone acetylation processes within somatic cell-descended chromatin lysine moieties among bovine cloned embryos. Finally, this leads to the increased in vitro developmental potential of cloned embryos in this large ruminant species (Ding et al., 2008; Iager et al., 2008). As a consequence, these investigators have proved that combined (i.e., 5-aza-dC- and TSA-dependent) epigenomic modulation of nuclear donor cells and early SCNT-derived embryos, was more efficient than those mediated by only single agents (either 5-aza-dC or TSA). Analogously to the results obtained in the research of the above-mentioned authors, the results of the study by Saini et al. (2017) have confirmed that the comparable beneficial effects can be achieved by exposing either buffalo nuclear donor cells or early SCNT-derived embryos or both of them to a combination of TSA and 5-aza-dC. Nonetheless, using a double treatment with these agents, these investigators have found that it is enough to epigenomically modulate only nuclear DNA of donor cells and, thus, there is no need of modulation of both nuclear donor cells and SCNT-derived embryos.

Summing up, the results of our study have shown that TSA-dependent epigenomic modulation of somatic cell-inherited nuclear genome improves the in vitro developmental competences of caprine cloned embryos. Two-step TSA treatment of donor cell nuclei (before being used for SCNT and following activation of NT oocytes) has contributed to enhancements in the morula and blastocyst formation rates. However, these enhancements are comparable to those achieved as a result of the exposure of only in vitro cultured nuclear donor cells to TSA. This suggests that artificial TSA-mediated modulation of the epigenetically regulated transcriptional activity of somatic cell-descended nuclear genome affects positively its reprogrammability in a host cytoplasm of activated NT oocytes and subsequently in a cytoplasm of descendant blastomeres of developing caprine SCNT-derived embryos. Moreover, it is worth highlighting that, so far, none of the studies have reported the application of TSA-dependent strategies aimed at epigenomic modulation of either nuclear donor cells and subsequently activated NT oocytes or only activated NT oocytes for the purposes of efficient generating goat embryos by somatic cell cloning. 


\section{References}

Agrawal H., Selokar N.L., Sa ini M., S ingh M.K., Chauhan M.S., Palta P., S ing la S.K., M a n ik R.S. (2018). Epigenetic alteration of donor cells with histone deacetylase inhibitor $m$-carboxycinnamic acid bishydroxymide improves the in vitro developmental competence of buffalo (Bubalus bubalis) cloned embryos. Cell. Reprogram., 20: 76-88.

Chi es J.M., P ole ja ev a I.A., R odrigues J.L., For ell F., B e rtolin i L.R., B e rtolini M. (2016). Developmental outcome and related abnormalities in goats: comparison between somatic cell nuclear transfer- and in vivo-derived concepti during pregnancy through term. Cell. Reprogram., 18: 264-279.

Deng M., Ren C., Liu Z., Z hang G., Wang F., Wan Y. (2017). Epigenetic status of H19-Igf2 imprinted genes and loss of 5-hydroxymethylcytosine in the brain of cloned goats. Cell. Reprogram., 19: 199-207.

D ing X., Wang Y., Z hang D., Wang Y., Gu o Z., Z hang Y. (2008). Increased pre-implantation development of cloned bovine embryos treated with 5-aza-2'-deoxycytidine and trichostatin A. Theriogenology, 70: 622-630.

Eilertsen K.J., P ow er R.A., Harkins L.L., Mis i c a P. (2007). Targeting cellular memory to reprogram the epigenome, restore potential, and improve somatic cell nuclear transfer. Anim. Reprod. Sci., 98: 129-146.

F a n Z., Yang M., R e g o u s k i M., P o le j a e v a I.A. (2019). Gene knockouts in goats using CRISPR/Cas9 system and somatic cell nuclear transfer. Methods Mol. Biol., 1874: 373-390.

Fernandes C.C.L., A gui a r L.H., Cald erón C.E.M., Silva A.M., A 1 ves J.P.M., Ros setto R., Bertolini L.R., Bertolini M., Rondina D. (2018). Nutritional impact on gene expression and competence of oocytes used to support embryo development and livebirth by cloning procedures in goats. Anim. Reprod. Sci., 188: 1-12.

Gupta M.K., He o Y.T., Kim D.K., L e e H.T., Uhm S.J. (2019). 5-Azacytidine improves the meiotic maturation and subsequent in vitro development of pig oocytes. Anim. Reprod. Sci., 208: 106118.

Hosseini S.M., Dufort I., Nieminen J., Moulavi F., Ghanaei H.R., Hajian M., Jafarpour F., Forouzanfar M., Gourbai H., Shahverdi A.H., Nasr-Esfahan i M.H., S ir a rd M.A. (2016). Epigenetic modification with trichostatin A does not correct specific errors of somatic cell nuclear transfer at the transcriptomic level; highlighting the non-random nature of oocyte-mediated reprogramming errors. BMC Genomics, 17: 16.

Huan Y., Wu Z., Zhang J., Zhu J., Liu Z., Song X. (2015). Epigenetic modification agents improve gene-specific methylation reprogramming in porcine cloned embryos. PLoS One, 10 (6): e0129803.

I a g e r A.E., R a gin a N.P., R o s s P.J., B e y han Z., Cunniff K., R odrigue z R.M., Cibe lli J.B. (2008). Trichostatin A improves histone acetylation in bovine somatic cell nuclear transfer early embryos. Cloning Stem Cells, 10: 371-379.

Jia R., Zhang G., F an Y., Zhou Z., Wan Y., Zhang Y., Wang Z., Wang F. (2017). MBD1 and MeCP2 expression in embryos and placentas from transgenic cloned goats. Zygote, 25: $462-471$.

J in J.X., Le e S., Taw e e ha ipa is a nkul A., Kim G.A., Le e B.C. (2017). The HDAC inhibitor LAQ824 enhances epigenetic reprogramming and in vitro development of porcine SCNT embryos. Cell. Physiol. Biochem., 41: 1255-1266.

Jin L., Guo Q., Zhang G.L., Xing X.X., Xuan M.F., Luo Q.R., Luo Z.B., Wang J.X., Y in X.J., Kang J.D. (2018). The histone deacetylase inhibitor, CI994, improves nuclear reprogramming and in vitro developmental potential of cloned pig embryos. Cell. Reprogram., 20: $205-213$.

Kishigami S., Mizutani E., Ohta H., Hikichi T., Thuan N.V., Wakayama S., B u i H.T., Wa k a y m a T. (2006). Significant improvement of mouse cloning technique by treatment with trichostatin A after somatic nuclear transfer. Biochem. Biophys. Res. Commun., 340: 183-189.

K u m a r D., S a rkhel B.C. (2017). Differential expression pattern of key regulatory developmental genes in pre-implant zona free cloned vs in vitro fertilized goat embryos. Gene Expr. Patterns, 25-26: 118-123. 
Liu Y., Wu F., Zhang L., Wu X., Li D., Xin J., Xie J., Kong F., Wang W., Wu Q., Zhang D., Wang R., Ga o S., Li W. (2018). Transcriptional defects and reprogramming barriers in somatic cell nuclear reprogramming as revealed by single-embryo RNA sequencing. BMC Genomics, 19: 734.

L o i P., I us o D., Czernik M., Ogura A. (2016). A new, dynamic era for somatic cell nuclear transfer? Trends Biotechnol., 34: 791-797.

L u R., Z hang T., Wu D., H e Z., J i a ng L., Z h o u M., C h e ng Y. (2018). Production of functional human CuZn-SOD and EC-SOD in bitransgenic cloned goat milk. Transgenic Res., 27: 343-354.

M a o T., Han C., D eng R., We i B., Meng P., L u o Y., Z hang Y. (2018). Treating donor cells with 2-PCPA corrects aberrant histone H3K4 dimethylation and improves cloned goat embryo development. Syst. Biol. Reprod. Med., 64: 174-182.

Martins L.T., Neto S.G., Tavares K.C., Calderón C.E., Aguiar L.H., Lazzarot to C.R., Ongaratto F.L., Rodrigues V.H., Carne iro Ide S., Ros s e t to R., A 1 me i d a A.P., F e rnandes C.C., R ond in a D., D i a s A.C., Chies J.M., P ol eja e va IA., R o drigues J.L., For ell F., B ertolin i L.R., B e rtolini M. (2016). Developmental outcome and related abnormalities in goats: comparison between somatic cell nuclear transfer- and in vivoderived concepti during pregnancy through term. Cell. Reprogram., 18: 264-279.

O p i e la J., S a m i e c M., R o m a n e k J. (2017). In vitro development and cytological quality of inter-species (porcine $\rightarrow$ bovine) cloned embryos are affected by trichostatin A-dependent epigenomic modulation of adult mesenchymal stem cells. Theriogenology, 97: 27-33.

Q i u X., You H., X i a o X., L i N., L i Y. (2017). Effects of trichostatin A and PXD101 on the in vitro development of mouse somatic cell nuclear transfer embryos. Cell. Reprogram., 19: 1-9.

Rodriguez-Os orio N., Urrego R., Cibelli J.B., Eilertsen K., Memili E. (2012). Reprogramming mammalian somatic cells. Theriogenology, 78: 1869-1886.

Saini M., Selokar N.L., Revey T., Singla S.K., Chauhan M.S., Palta P., Madan P. (2014). Trichostatin A alters the expression of cell cycle controlling genes and microRNAs in donor cells and subsequently improves the yield and quality of cloned bovine embryos in vitro. Theriogenology, 82: 1036-1042.

S a in i M., S e lo kar N.L., A grawal H., S ingla S.K., Chauhan M.S., Manik R.S., Pal t a P. (2017). Treatment of donor cells and reconstructed embryos with a combination of trichostatin-A and 5-aza-2'-deoxycytidine improves the developmental competence and quality of buffalo embryos produced by handmade cloning and alters their epigenetic status and gene expression. Cell. Reprogram., 19: 208-215.

S a m i e c M. (2004). Development of pig cloning studies: past, present and future. J. Anim. Feed Sci., 13: $211-238$.

S a m i e c M., S krzy s z o w s k a M. (2010). Preimplantation developmental capability of cloned pig embryos derived from different types of nuclear donor somatic cells. Ann. Anim. Sci., 10: 385-398.

S a m i e c M., S krzys zow s k M. (2011). Transgenic mammalian species, generated by somatic cell cloning, in biomedicine, biopharmaceutical industry and human nutrition/dietetics - recent achievements. Pol. J. Vet. Sci., 14: 317-328.

S a m i e c M., S krzy s z ow s k a M. (2012). High developmental capability of porcine cloned embryos following trichostatin A-dependent epigenomic transformation during in vitro maturation of oocytes pre-exposed to $R$-roscovitine. Anim. Sci. Pap. Rep., 30: 383-393.

S a m i e c M., S krzy s z o w s k a M. (2018 a). Can reprogramming of overall epigenetic memory and specific parental genomic imprinting memory within donor cell-inherited nuclear genome be a major hindrance for the somatic cell cloning of mammals? - a review. Ann. Anim. Sci., 18: 623-638.

S a m i e c M., S krzy s z o w s k a M. (2018 b). Intrinsic and extrinsic molecular determinants or modulators for epigenetic remodeling and reprogramming of somatic cell-derived genome in mammalian nuclear-transferred oocytes and resultant embryos. Pol. J. Vet. Sci., 21: 217-227.

S a m i e c M., O p i e l a J., L i p ińs k i D., R o m a n e k J. (2015). Trichostatin A-mediated epigenetic transformation of adult bone marrow-derived mesenchymal stem cells biases the in vitro developmental capability, quality, and pluripotency extent of porcine cloned embryos. Biomed Res. Int., 2015: 814686.

S a m i e c M., R o man ek J., L i p iń s k i D., O p i e la J. (2019). Expression of pluripotency-related genes is highly dependent on trichostatin A-assisted epigenomic modulation of porcine mesenchy- 
mal stem cells analysed for apoptosis and subsequently used for generating cloned embryos. Anim. Sci. J., 90: 1127-1141.

S angalli J.R., Chiaratti M.R., D e B em T. H., de Araújo R.R., Bress a n F.F., S a mp a i o R.V., P e re c in F., S m ith L.C., K ing W.A., M e ire lle s F.V. (2014). Development to term of cloned cattle derived from donor cells treated with valproic acid. PLoS One, 9 (6): e101022.

Sepulveda-Rincon L.P., Solanas Edel L., Serrano-Revuelta E., Ruddick L., M a a louf W.E., B e a u j e a n N. (2016). Early epigenetic reprogramming in fertilized, cloned, and parthenogenetic embryos. Theriogenology, 86: 91-98.

Wan Y., Deng M., Zhang G., Ren C., Zhang H., Zhang Y., Wang L., Wang F. (2016). Abnormal expression of DNA methyltransferases and genomic imprinting in cloned goat fibroblasts. Cell Biol. Int., 40: 74-82.

Wang Y.S., Xi ong X.R., An Z.X., Wang L.J., Li u J., Qu an F.S., Hua S., Zhang Y. (2011). Production of cloned calves by combination treatment of both donor cells and early cloned embryos with 5-aza-2'-deoxycytidine and trichostatin A. Theriogenology, 75: 819-825.

Wang Y.M., D ing X.B., L i u X.F., Z h a n g Y. (2015). Donor cell trichostatin A treatment improves the in vitro development of cloned goat embryos. Small Ruminant Res., 124: 76-80.

We n B.Q., L i J., L i J.J., T i an S.J., S u n S.C., Q i X., C a i W.T., C h a n g Q.L. (2014). The histone deacetylase inhibitor Scriptaid improves in vitro developmental competence of ovine somatic cell nuclear transferred embryos. Theriogenology, 81: 332-339.

Z hang Y.L., Z hang G.M., J i a R.X., Wan Y.J., Yang H., S u n L.W., H a n L., Wang F. (2018). Non-invasive assessment of culture media from goat cloned embryos associated with subjective morphology by gas chromatography - mass spectroscopy-based metabolomic analysis. Anim. Sci. J., 89: 31-41.

Zu o Y., S u G., Cheng L., Li u K., F eng Y., We i Z., B a i C., C a o G., Li G. (2017). Coexpression analysis identifies nuclear reprogramming barriers of somatic cell nuclear transfer embryos. Oncotarget, 8: 65847-65859.

Received: 15 V 2019

Accepted: 12 IX 2019 